\title{
Electrostatically Confined Monolayer Graphene Quantum Dots with Orbital and Valley Splittings
}

Nils M. Freitag, ${ }^{\dagger}$ Larisa A. Chizhova, $^{\dagger}$ Peter Nemes-Incze, ${ }^{\dagger}$ Colin R. Woods, ${ }^{\text {II }}$ Roman V. Gorbachev, ${ }^{\text {If }}$ Yang Cao, ${ }^{\text {I }}$ Andre K. Geim, ${ }^{\text {II }}$ Kostya S. Novoselov, ${ }^{\text {If }}$ Joachim Burgdörfer, ${ }^{\dagger}$ Florian Libisch, ${ }^{\dagger}$ and Markus Morgenstern ${ }^{*} \dagger$

${ }^{\dagger}$ II. Institute of Physics B and JARA-FIT, RWTH Aachen University, Otto-Blumenthal-Straße, 52074 Aachen, Germany

${ }^{\ddagger}$ Institute for Theoretical Physics, TU Wien, Wiedner Hauptstraße 8-10, 1040 Vienna, Austria, EU

${ }^{\text {II }}$ School of Physics and Astronomy, University of Manchester, Manchester M13 9PL, United Kingdom

\section{Supporting Information}

ABSTRACT: The electrostatic confinement of massless charge carriers is hampered by Klein tunneling. Circumventing this problem in graphene mainly relies on carving out nanostructures or applying electric displacement fields to open a band gap in bilayer graphene. So far, these approaches suffer from edge disorder or insufficiently controlled localization of electrons. Here we realize an alternative strategy in monolayer graphene, by combining a homogeneous magnetic field and electrostatic confinement. Using the tip of a scanning tunneling microscope, we induce a confining potential in the Landau gaps of bulk graphene without the need for physical

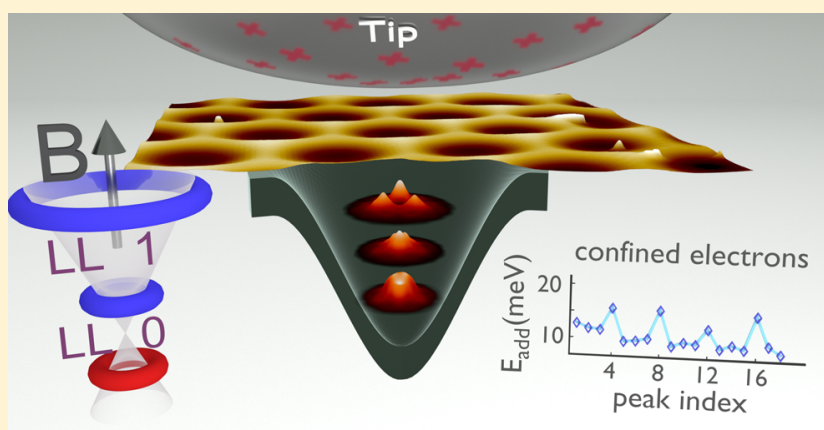
edges. Gating the localized states toward the Fermi energy leads to regular charging sequences with more than 40 Coulomb peaks exhibiting typical addition energies of 7-20 meV. Orbital splittings of 4-10 meV and a valley splitting of about $3 \mathrm{meV}$ for the first orbital state can be deduced. These experimental observations are quantitatively reproduced by tight binding calculations, which include the interactions of the graphene with the aligned hexagonal boron nitride substrate. The demonstrated confinement approach appears suitable to create quantum dots with well-defined wave function properties beyond the reach of traditional techniques.

KEYWORDS: Graphene, quantum dot, valley splitting, orbital splitting, STM, Landau quantization

$\mathrm{T}$ he charge carriers in graphene at low energies, described as massless Dirac quasiparticles, ${ }^{1}$ are expected to feature long spin coherence times. ${ }^{2-5}$ Exploiting this property requires precise manipulation of individual Dirac electrons. Quantum dots (QDs) present an essential building block, yet providing tailored confinement in graphene has remained challenging. So far, e-beam lithography ${ }^{6}$ and various other techniques ${ }^{7-12}$ have been used to design nanometer-sized devices. However, their performance lacks behind, for example, GaAs QDs, ${ }^{13,14}$ as disordered sample edges of patterned graphene result in uncontrolled charge localization and scattering. ${ }^{6,15-17}$ So far, no clear evidence for 4-fold degenerate charging sequences has been reported in transport measurements of tunable QDs. Moreover, failing to controllably lift graphene's valley degeneracy renders spin qubits unfeasible. ${ }^{2,18,19}$

In principle, bilayer graphene could improve the situation because an electric displacement field opens a band gap at regular $\mathrm{AB}$ stacking. ${ }^{20}$ Indeed, electrostatically confined QDs in bilayer graphene exhibit Coulomb blockade, ${ }^{21-23}$ yet controlling the spin or valley degree of freedom of an individual state has also not been demonstrated. Moreover, confinement is still prone to parasitic conduction channels due to residual disorder in the band gap or conducting channels along domain walls of AB- and BA-stacked areas. ${ }^{24}$ Another approach exploits whispering gallery modes in electrostatically confined $\mathrm{QDs}^{25-27}$ but here the control of the wave functions by gates is difficult and dwell times are extremely short ( $<100 \mathrm{fs})$. On an even more intricate route, the tip of a scanning tunneling microscope (STM) is used to locally stretch a suspended monolayer graphene sheet. ${ }^{28}$ The onset of charge quantization due to induced strain showcases confinement by pseudomagnetic fields. Adding a real magnetic field $B$ leads to charging sequences with regular orbital but no valley splittings. ${ }^{28}$ Creating multiple QDs in this fashion would require independent strain control for every $\mathrm{QD}$ on the suspended graphene. Thus, such an approach is barely scalable.

Landau quantization helps to overcome Klein tunneling by opening band gaps. ${ }^{21-23}$ An elegant method to exploit this by combining a magnetic field and an electrostatic potential has been proposed theoretically. ${ }^{29-31}$ Indeed, indications of such

Received: June 21, 2016

Revised: July 22, 2016

Published: July 28, 2016 

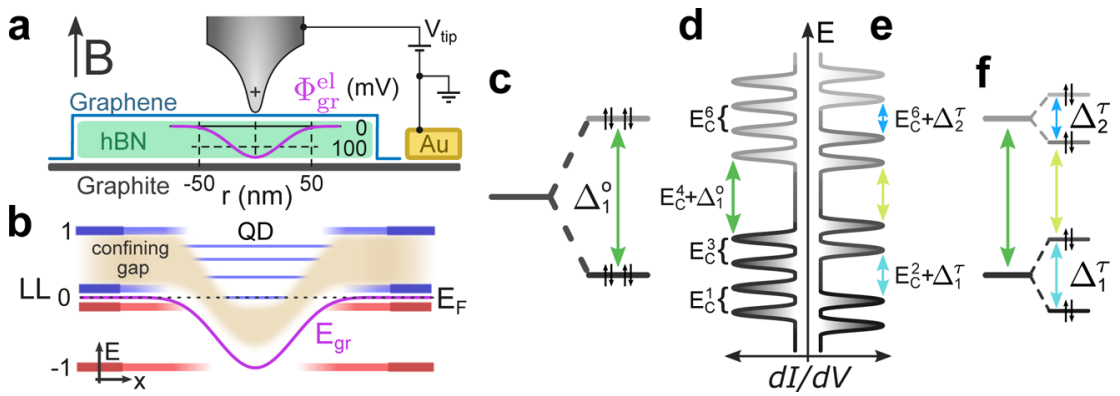

Figure 1. (a) Sketch of the experiment. Graphene covers a $30 \mathrm{~nm}$ thick hexagonal boron nitride flake on graphite. The magenta line represents the tip-induced confinement potential of graphene $\Phi_{\mathrm{gr}}^{\mathrm{el}}$ for electrons, calculated as the numerical solution of Poisson's equation (Supporting Information). (b) Energy diagram in real space: Fermi energy $E_{\mathrm{F}}$, black dashed line; local band bending $E_{\mathrm{gr}}$, magenta line; states belonging to electron (hole) LLs, blue (red); bulk LLs, 1, 0, -1. States embedded in the $\mathrm{LL}_{0}-\mathrm{LL}_{+1}$ gap (thin blue lines) are electrostatically confined. (c) Energy level diagram for the first two orbital states of a graphene QD exhibiting an orbital splitting $\Delta_{1}^{\circ}$. Both orbitals are 4-fold degenerate, as indicated by black arrows representing physical spin. (d,e) Charging peak sequence in the differential conductance $\mathrm{d} I / \mathrm{d} V$ corresponding to the level diagrams in panels $\mathrm{c}$ and $\mathrm{f}$, respectively. Charging peaks are separated by the addition energy $E_{\text {add }}^{i}=E_{\mathrm{C}}^{i}+\Delta_{i}$ where $E_{\mathrm{C}}^{i} \approx E_{\mathrm{C}}$ is the charging energy and $\Delta_{i}$ is comprised of $\Delta_{j}^{\mathrm{o}}$ and/or the valley splittings $\Delta_{k}^{\tau}$. In panel d, quadruplet ordering showcases a dominant $\Delta_{1}^{\mathrm{o}}$, while $\Delta_{k}^{\tau}$ become sizable in panel e, further separating quadruplets into doublets. (f) Same as panel c but including additional $\Delta_{k}^{\tau}$. The spin splitting $\Delta^{\sigma}$ is neglected, as $\Delta^{\sigma}<\Delta_{k}^{\tau}, \Delta_{j}^{\circ}, E_{\mathrm{C}}$ in experiment.

confinement have been found in metal contact-induced pnp junctions, ${ }^{32}$ graphene on $\mathrm{SiO}_{2},{ }^{33,34}$ and a suspended graphene nanoribbon. ${ }^{35}$ However, in these experiments the confinement potential was not tunable but was generated by electrostatic disorder.

Here, we demonstrate controlled confinement by a combination of magnetic and electrostatic fields. We use the tip-induced electrostatic potential of an $\mathrm{STM}^{36,37}$ in a $B$ field perpendicular to the graphene plane (Figure 1a). Scanning tunneling spectroscopy (STS) reveals sequences of charging peaks by means of Coulomb staircases that appear when these confined states cross the Fermi energy $E_{\mathrm{F}}$. The peaks systematically group in quadruplets for electrons and holes corresponding to the 4-fold (valley and spin) degeneracy in graphene (Figure 1c,d). Moreover, some quadruplets separate into doublets due to an additional valley splitting induced by the hexagonal boron nitride (BN) substrate. STS as a function of $B$ reveals that the first confined states emerge from Landau levels (LLs) with indices \pm 1 . A third-nearest neighbor tight binding (TB) calculation ${ }^{38,39}$ reproduces the onset of charging events as a function of tip voltage $V_{\text {tip }}$ and $B$ as well as the magnitude of orbital and valley splittings.

We now sketch the principle of our experiment. A homogeneous, perpendicular $B$ field condenses the electronic states of graphene into LLs at energies

$$
E_{N}=\operatorname{sgn}(N) \sqrt{2 \hbar e \nu_{\mathrm{F}}^{2}|B N|}
$$

where $\nu_{\mathrm{F}}$ is the Fermi velocity and $N \in \mathbb{Z}$ is the LL index. ${ }^{1}$ Consequently, energy gaps between the LLs emerge in the electronic spectrum. The smooth electrostatic potential $\Phi_{\mathrm{gr}}^{\mathrm{el}}$ (magenta line in Figure 1a) induced by the STM tip locally shifts the eigenenergies $\varepsilon_{i}\left(\Phi_{\mathrm{gr}}^{\mathrm{el}}\right)$ of charge carriers relative to the bulk LL energy (eq 1$)$. Shifting $\varepsilon_{i}$ into the Landau gaps creates confined states (Figure $1 \mathrm{~b}$ ). ${ }^{30}$ The shape of $\Phi_{\mathrm{gr}}^{\mathrm{el}}$ determines the single-particle orbitals and energy levels, as in the case of artificial atoms. ${ }^{14}$ Orbital splittings $\Delta_{j}^{\mathrm{o}}$ separate the energy levels (Figure 1c), which we deduce experimentally to be $\Delta_{j}^{o}=4-10$ $\mathrm{meV}$ (see below) and thus $\Delta_{j}^{\mathrm{o}}$ is small compared to the first LL gap $E_{1}-E_{0} \approx 100 \mathrm{meV}$ at $7 \mathrm{~T}$. While pristine graphene exhibits a 4-fold degeneracy, varying stacking orders of graphene on top of $\mathrm{BN}$ induce an additional valley splitting
$\Delta_{k}^{\tau}$, which turns out to be smaller than $\Delta_{j}^{\mathrm{o}}$ in our experiment. The finite $B$ field creates a small Zeeman splitting estimated as $\Delta^{\sigma}=g \mu_{\mathrm{B}} B \approx 800 \mu \mathrm{eV}$ at $7 \mathrm{~T}$ ( $g$-factor of $2, \mu_{\mathrm{B}}$ : Bohr's magneton). Accordingly, the orbital splittings separate quadruplets of near-degenerate $\mathrm{QD}$ states, which exhibit a subtle spin-valley substructure (Figure 1f).

We use the STM tip not only as source of the electrostatic potential and thus as gate for the $\mathrm{QD}$ states but also to sequence the energy level spectrum of the $\mathrm{QD}$ as the states cross $E_{\mathrm{F}}$, that is, as the charge on the $\mathrm{QD}$ changes by $\pm e$. This leads to a step in the tunneling current $I\left(V_{\text {tip }}\right)$ and a corresponding charging peak in the differential conductance $\mathrm{d} I / \mathrm{d} V_{\text {tip }}$. In addition to the single particle energy spacings, every additional electron on the dot needs to overcome the electrostatic repulsion to the electrons already inside the $\mathrm{QD},{ }^{40}$ given by the charging energy $E_{\mathrm{C}}^{i}$. Thus, we probe the total energetic separation of charge states $i$ and $i+1$, given by the addition energy $E_{\text {add }}^{i}=E_{\mathrm{C}}^{i}+\Delta_{i}$, where $\Delta_{i}$ consists of $\Delta_{j}^{o}, \Delta_{k}^{\tau}$, and/or $\Delta^{\sigma}$. As we experimentally find $E_{\mathrm{C}}^{i} \approx E_{\mathrm{C}} \approx 10 \mathrm{meV} \gtrsim \Delta_{j}^{\mathrm{o}}$ (nearly independent of the charge state $i$, see below), the quadruplet near-degeneracy of the QD states translates to quadruplet ordering of the charging peaks (Figure 1d). Whenever either $\Delta_{k}^{\tau}$ or $\Delta^{\sigma}$ significantly exceeds the other and temperature, quadruplets separate into doublets (Figure 1e).

We prepare our sample (see Figure la and Supporting Information) by dry-transferring ${ }^{41,42}$ a graphene flake onto BN. ${ }^{43-45}$ During this step, we align both crystal lattices with a precision better than $1^{\circ}$ (Supporting Information). Then we place this graphene/BN stack on a large graphite flake to avoid insulating areas and simplify navigating the STM tip. Any disorder potential present in the sample will limit the confinement as long as it is larger than the Landau level gaps, thus larger gaps (e.g., the $\mathrm{LL}_{0}-\mathrm{LL}_{ \pm 1}$ gap) result in improved confinement. Moreover, the induced band bending will only be well-defined if the disorder potential is smaller than the maximum of $\Phi_{\mathrm{gr}}^{\mathrm{el}}$. By using the dry-transfer technique ${ }^{41,42}$ and a graphite/BN substrate we reduce disorder in the graphene significantly. ${ }^{46-48}$

Probing the sample in our custom-build UHV-STM system ${ }^{49}$ at $T=8 \mathrm{~K}$, we observe the superstructure with $a=13.8 \mathrm{~nm}$ periodicity, which develops due to the small lattice mismatch of $1.8 \%$ between graphene and $\mathrm{BN} .^{47}$ An atomically resolved STM 


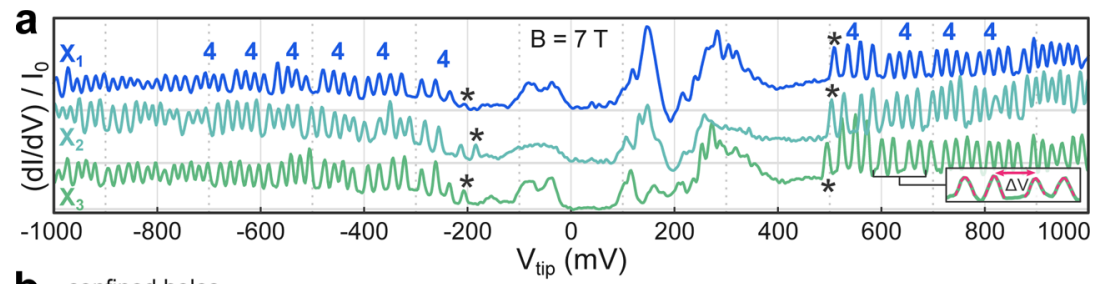

b

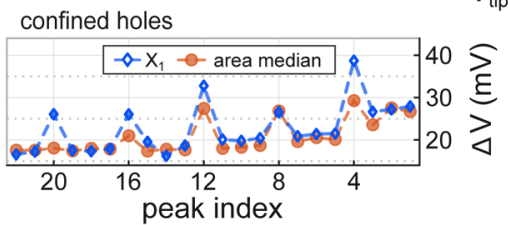

C
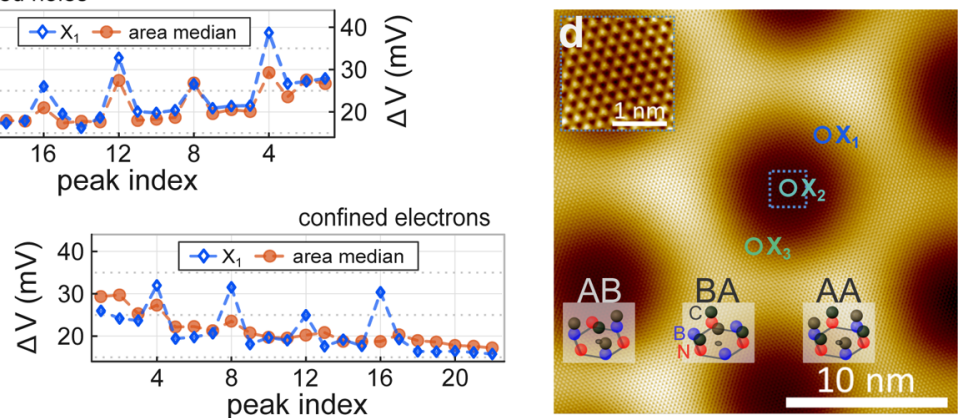

Figure 2. (a) Representative differential conductance spectra $\mathrm{d} I / \mathrm{d} V\left(V_{\text {tip }}\right)$, normalized by the first value $I_{0}$ of the respective $I\left(V_{\text {tip }}\right)$ curve (Supporting Information). Recording positions are $\mathrm{X}_{1}$, between $\mathrm{AA}$ and $\mathrm{AB} ; \mathrm{X}_{2}$, on $\mathrm{AB} ; \mathrm{X}_{3}$, between $\mathrm{AB}$ and $\mathrm{BA}$ (compare panel $\mathrm{d}$ ). Spectra on other regions (e.g., AA, BA) look similar. $V_{\text {stab }}=1 \mathrm{~V}, I_{\text {stab }}=700 \mathrm{pA}, V_{\text {mod }}=4.2 \mathrm{mV}_{\text {rms }}$ and $B=7 \mathrm{~T}$. Quadruplets of peaks are marked by "4" and the first charging peak on either $V_{\text {tip }}$ side by an asterisk. Curves are offset for clarity, while horizontal gray lines mark $\mathrm{d} I / \mathrm{d} V=0 \mathrm{~S}$. Inset shows a zoom with Gaussian fits (dashed lines) used to extract distances between adjacent peaks $\Delta V$ as marked. $(\mathrm{b}, \mathrm{c}) \Delta V$ as a function of consecutive peak index for spectrum $\mathrm{X}_{1}$ (blue, error bars smaller than symbol size) and the median values for $80 \times 80$ spectra recorded on $60 \times 60 \mathrm{~nm}^{2}$ (orange). (d) Atomically resolved STM image (raw data) of the aligned graphene on hexagonal boron nitride (BN). $V_{\text {tip }}=400 \mathrm{mV}, I=1 \mathrm{nA}$. Differently stacked areas AB, BA, and AA marked and sketched by ball models. Inset on the upper left shows a zoom into the $A B$ stacked area, marked by the blue square, exhibiting an obvious sublattice symmetry breaking due to the underlying BN. Positions equivalent to those where spectra in panel a were recorded are marked by circles labeled $\mathrm{X}_{1}, \mathrm{X}_{2}, \mathrm{X}_{3}$.

image of this superstructure is presented in Figure 2d. Prior to measuring $\mathrm{d} I / \mathrm{d} V$ spectra, the tip-sample distance is adjusted at the stabilization voltage $V_{\text {stab }}$ and current $I_{\text {stab }}$ and then the feedback loop is turned off (Supporting Information). Figure 2a shows exemplary $\mathrm{d} I / \mathrm{d} V$ spectra, acquired at $B=7 \mathrm{~T}$ and adjusted to the same vertical scale by dividing $\mathrm{d} I / \mathrm{d} V$ by the first value $I_{0}$ of the respective $I(V)$ curve (Supporting Information). We observe pronounced, regularly spaced peaks for $V_{\text {tip }}<-170$ $\mathrm{mV}$ and $V_{\text {tip }}>500 \mathrm{mV}$. A closer look at the sequences reveals the expected grouping in quadruplets, which can still be distinguished up to the 20th peak. This grouping becomes even more evident by directly comparing the voltage difference between adjacent peaks $\Delta V$ in Figure $2 \mathrm{~b}, \mathrm{c} ; \Delta V$ between quadruplets is up to twice as large as $\Delta V$ within the quadruplets indicating $\Delta_{j}^{\mathrm{o}} \lesssim E_{\mathrm{C}}^{i}$ while $\Delta_{k}^{\tau}$ and $\Delta^{\sigma}$ are significantly smaller. To further elucidate grouping patterns, we measure $6400 \mathrm{~d} I / \mathrm{d} V$ spectra at equidistant positions within a $60 \mathrm{~nm} \times 60 \mathrm{~nm}$ area, thus probing all areas of the superstructure. The median $\Delta V$ values (orange circles in Figure $2 b, c$ ) portray the robust ordering into quadruplets on the hole side, implying $\Delta_{j}^{o}$ generally dominates over $\Delta_{k}^{\tau}$ and $\Delta^{\sigma}$. On the electron side of the spectra, the sequences are disturbed by a few additional charging peaks of defect states in the $\mathrm{BN}$ substrate ${ }^{50}$ that are identified by their characteristic spatial development (Supporting Information). This limits the comparability of the electron and hole sector and hides possible smaller electron-hole asymmetries in the data. The $\mathrm{d} I / \mathrm{d} V$ features in between the charging peaks most likely capture contributions from multiple orbital states of each LL, which are lifted in degeneracy by the tip-induced potential, but cannot be identified unambiguously (Supporting Information, Section 5).

To understand the origin of the charging peaks, we provide a detailed microscopic picture of the tip-induced gating of localized states. We will only discuss the case of positive $V_{\text {tip }}$, that is, electron confinement, because the arguments for negative $V_{\text {tip }}$ are analogous. Increasing $V_{\text {tip }}$ (orange arrow in Figure 3) shifts the states underneath the tip energetically down. States originating from LLs with positive index are embedded in the $\mathrm{LL}_{0}-\mathrm{LL}_{+1}$ gap that provides electrostatic confinement (Figure 3a, see also Figure 1b). Within the bias window $e V_{\text {tip }}=\mu_{\text {gr }}-\mu_{\text {tip }}$, electrons tunnel from the sample into unoccupied states of the tip. One current path (dashed green arrow Figure 3a) passes through states of the QD (blue lines). The other stronger current path (solid green arrow Figure 3a) originates from the quasi-continuous LDOS at lower energies where energetically overlapping LL states strongly couple to the graphene bulk. Though increasing $V_{\text {tip }}$ gates QD states down (Figure $3 \mathrm{~b}$ ), the Coulomb gap around $E_{\mathrm{F}}$ always separates the highest occupied from the lowest unoccupied state, prohibiting continuous charging of confined states. It is only when the next unoccupied level crosses $\mu_{\mathrm{gr}}$ that the $\mathrm{QD}$ is charged by an additional electron. The electrostatic repulsion due to its charge abruptly increases the Hartree energy of all states, thereby shifting additional graphene states from below $\mu_{\text {tip }}$ into the bias window (Figure 3b, central transition). Consequently, the tunneling current $I$ increases which translates to a charging peak in $\mathrm{d} I / \mathrm{d} V_{\text {tip }}$ (Figure $3 \mathrm{c}$ ). This mechanism is called Coulomb staircase ${ }^{40}$ and has been observed previously, for instance, for charging of clusters within an STM experiment. ${ }^{51}$ In essence, charging peaks in $\mathrm{d} I / \mathrm{d} V$ signal the coincidence of a charge level of the QD with $\mu_{\mathrm{gr}}^{52}$ and thus provide a clear signature of the addition energy spectrum of the QD.

Because the measurement captures the $\mathrm{QD}$ level spacings as charging peak distances $\Delta V$, they need to be converted to $E_{\text {add }}$ via the tip lever arm $\alpha_{\text {tip }}$. The latter relates a change of $V_{\text {tip }}$ to its induced shift of the QD state energies. The lever arm is 


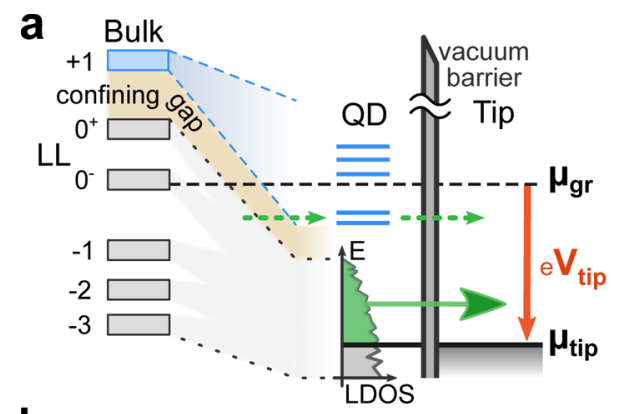

b

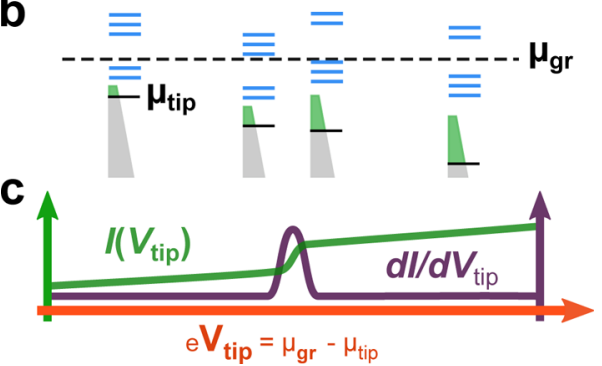

Figure 3. Sketch of the Coulomb staircase. (a) The chemical potentials of graphene $\mu_{\mathrm{gr}}$ (black dashed line) and tip $\mu_{\text {tip }}$ (black solid line) define the bias window $e V_{\text {tip }}$ within which graphene states tunnel into empty tip states. There are two current paths available: (i) a weak one (green dashed arrow) via quantum dot states (blue lines), (ii) a dominant one (solid green arrow) via states strongly coupled to the graphene bulk (marked LDOS). Left: bulk graphene LLs away from the tip-induced band bending. (b) Schematic diagram of change in QD energies (blue lines) and quasi-continuous LDOS underneath the tip (green and gray triangle) for increasing $V_{\text {tip }}$ from left to right. Between the second and third frame, the QD changes its charge state shifting the energy of the QD states and the entire LDOS upward. (c) Tunneling current $I$ displaying the staircase (green line) and differential conductance $\mathrm{d} I / \mathrm{d} V$ (purple line) for increasing $V_{\text {tip }}$ (aligned with panel b).

determined by the ratio of the capacitance between tip and dot $C_{\text {tip }}$, and the total capacitance of the $\operatorname{dot} C_{\Sigma}$, thus $\alpha_{\text {tip }}=C_{\text {tip }} / C_{\Sigma}$. $C_{\Sigma}$ includes $C_{\text {tip }}$, the capacitance between dot and back-gate, and dot and surrounding graphene. We use a Poisson solver to estimate $C_{\Sigma}=16.5 \pm 3.2 \mathrm{aF}$ and $C_{\text {tip }}=8 \pm 1.5 \mathrm{aF}$ for our QD (Supporting Information). Hence, we find $E_{\mathrm{C}}=e^{2} / C_{\Sigma} \approx 10 \pm 2$ $\mathrm{meV}$ and $\alpha_{\text {tip }}=0.51 \pm 0.03$ (close to values reported for a similar system by Jung et al. $^{33}$ ). Consequently charging peaks dominantly separated by $E_{\mathrm{C}}$, that is, $E_{\text {add }}^{i} \approx E_{\mathrm{C}}^{i}$ because $\Delta_{i} \ll$ $E_{\mathrm{C}}^{i}$, should exhibit $\Delta V=E_{\mathrm{C}} /\left(e \alpha_{\text {tip }}\right) \approx 20 \mathrm{mV}$, which is in close agreement with the values found within quadruplets at higher occupation numbers (Figure $2 \mathrm{~b}, \mathrm{c}$ ). As expected, we also find significantly larger $E_{\text {add }}^{i}$ for every fourth charging peak. In the case of clear quadruplet ordering, the orbital splittings for our $\mathrm{QD}$ are deduced from $\Delta_{j}^{o}=E_{\text {add }}^{4 j}-E_{\mathrm{C}}^{4 j} \approx E_{\text {add }}^{4 j}-E_{\text {add }}^{4 j+1}$ and we find typical values of 4-10 meV for the first few orbitals $\left(\alpha_{\text {tip }}=\right.$ 0.51 , Figure $2 b, c)$. For this estimate, we neglect the additional Zeeman splitting or an even smaller valley splitting.

We next provide a theoretical framework to elucidate the details of the QD level spectrum. The eigenstates of bulk graphene LLs (eq 1) feature different wave function amplitudes on sublattices ${ }^{1} \mathrm{~A}$ and $\mathrm{B}$

$$
\Psi_{N}^{\mathrm{K}}=\left(\begin{array}{c}
\Psi_{|N|-1}^{\mathrm{A}} \\
\Psi_{|N|}^{\mathrm{B}}
\end{array}\right) \quad \text { and } \quad \Psi_{N}^{\mathrm{K}^{\prime}}=\left(\begin{array}{c}
\Psi_{|N|}^{\mathrm{A}} \\
\Psi_{|N|-1}^{\mathrm{B}}
\end{array}\right)
$$

where $\mathrm{K}$ and $\mathrm{K}^{\prime}$ denote the two inequivalent $\mathrm{K}$-points of the Brillouin zone associated with the two valleys. For $N \neq 0$, the LL index differs by one for the two sublattices, while for $N=0$ the part of the wave function with subscript $|N|-1$ vanishes, resulting in polarized sublattices for each valley. The wave functions of bulk graphene (eq 2) are modified by the tipinduced potential. Assuming a radially symmetric confinement potential, the eigenstates are described by radial and angular momentum quantum numbers $\left(n_{\mathrm{r}}, m\right)$, with $n_{\mathrm{r}} \in \mathbb{N}_{0}$ and $m \in \mathbb{Z}$. Adiabatically mapping a given LL with index $N$ on to possible combinations of $n_{\mathrm{r}}$ and $m$ yields ${ }^{53}$

$$
|N|=n_{\mathrm{r}}+\frac{1}{2}(m+|m|)
$$

with $0 \leq n_{\mathrm{r}} \leq|N|$ and $m \leq|N|$.

We calculate eigenstates of a $120 \mathrm{~nm} \times 100 \mathrm{~nm}$ commensurate graphene flake on $\mathrm{BN}$ using third-nearest neighbor $\mathrm{TB},{ }^{38}$ where the substrate interaction enters via a periodic superstructure potential and local strain effects, ${ }^{39}$ parametrized from DFT calculations. ${ }^{54,55}$ We approximate the amplitude $\Phi_{0}^{\mathrm{el}}$ and shape of $\Phi_{\mathrm{gr}}^{\mathrm{el}}$ by a classic electrostatic solution of Poisson's equation (Figure 1a, Supporting Information) with the tip radius $r_{\text {tip }}$ as fit parameter. Comparing calculated charging energies to experiment yields a plausible value of $r_{\text {tip }} \approx 120 \mathrm{~nm}$, implying a full width at half-maximum (fwhm) of the QD confinement potential of $55 \mathrm{~nm}$ at $7 \mathrm{~T}$. We independently determine the initially free parameter $E_{\mathrm{F}}$ from the position of $\mathrm{LL}_{0}$ in STS as $E_{\mathrm{F}}=-40 \pm 5 \mathrm{meV}$ (Supporting Information). Accordingly, the graphene is p-doped. We note that varying $E_{\mathrm{F}}$ within the stated uncertainty range (see blue horizontal bar in Figure 4a) leads to no qualitative changes in the predictions of our model. We use open boundary conditions to simulate the coupling of the flake to the surrounding graphene. Consequently, eigenstates will feature complex eigenvalues $E_{l}=\varepsilon_{l}+i \Gamma_{l} / 2$, where the real part $\varepsilon_{l}$ represents the resonant energies and the imaginary part $\Gamma_{l}$ the coupling to the delocalized bulk states. ${ }^{56}$ Thus, we can readily distinguish states that are spread out over the flake (large $\Gamma_{l}$ ) from those localized near the tip $\left(\right.$ small $\left.\Gamma_{l}\right)$. We color code $\Gamma_{l}$ in Figure $4 \mathrm{a}$ for a calculation with the tip-induced potential centered on an $\mathrm{AB}$ stacked area.

At $B=7 \mathrm{~T}$ and vanishing band bending $\left(\Phi_{0}^{\mathrm{el}}=0\right)$, we find only delocalized states whose eigenenergies cluster around the bulk LL energies (eq 1, Figure 4a). As we increase $\Phi_{0}^{\mathrm{el}}$, states begin to localize at the tip and shift in energy, with smaller $\Gamma_{l}$ (darker curves) pointing to stronger localization (see Figure $4 a$ ). Comparing hole states originating from $\mathrm{LL}_{-1}$ for negative and positive $\Phi_{0}^{\mathrm{el}}$, we find, as expected, stronger localization in case of negative $\Phi_{0}^{\mathrm{el}}$. The potential is always attractive to one kind of charge carriers that will localize underneath the tip. The other kind is repelled by the induced potential (see also ref 31) which results in stronger coupling to the bulk. In order to classify our TB wave functions in terms of the quantum numbers $N, n_{\mathrm{r}}$, and $m$, we consider sublattice $\mathrm{A}$ and $\mathrm{B}$ separately. Tracing the states back to their LL of origin reveals $N$, constraining possible $n_{\mathrm{r}} \leq|N|$. The value of $n_{\mathrm{r}}$ is then determined by counting radial minima in the line cuts of the wave function amplitude for each sublattice (Figure $4 b-d$ ). The distance of the first radial maximum from the center of the wave function is finally sufficient to assign the possible $m$ quantum numbers of the LL (eq 3). Additionally, the $\left(n_{\mathrm{r}}, m\right)$ combinations need to be consistent with $N$ differing by one on the two sublattices (eq 2). For instance, the line cuts in Figure 


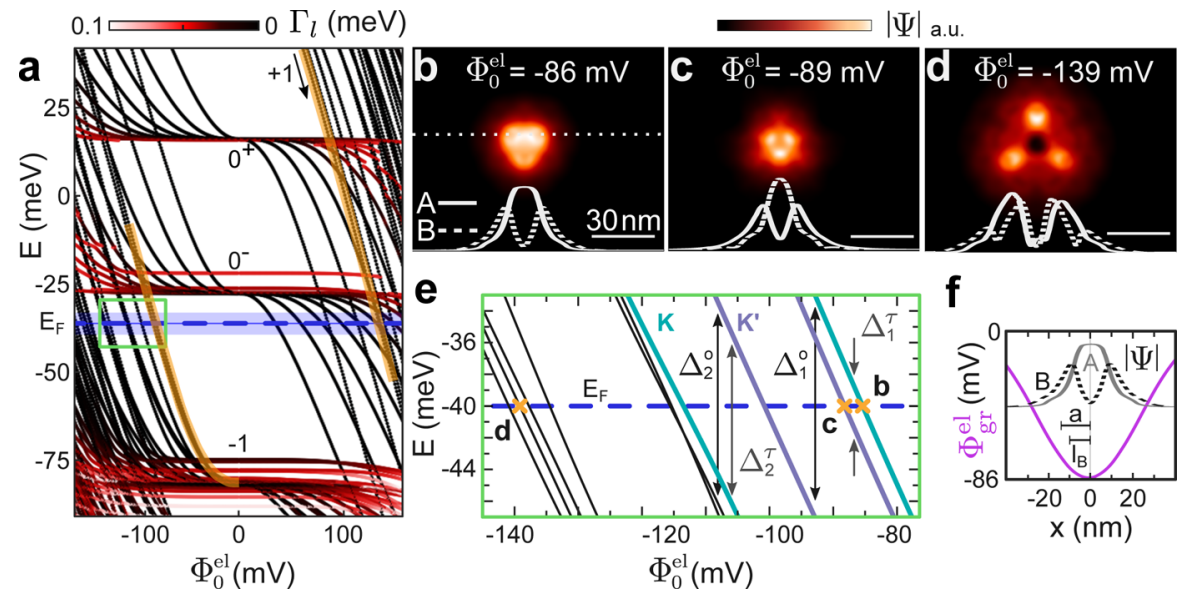

Figure 4. (a) Tight binding eigenenergies of a $120 \times 100 \mathrm{~nm}^{2}$ graphene sample with open boundaries as a function of tip-induced potential amplitude $\Phi_{0}^{\text {el }}$ at $B=7 \mathrm{~T}$ with the tip-induced potential centered on an AB area (BA and AA yield very similar behavior, not shown). Line color encodes coupling to the boundary (imaginary part $\Gamma_{l}$ of eigenenergies); black (red) indicates strong (weak) localization underneath the tip. States from $L_{ \pm 1}$ and the split $L_{0}$ are labeled by \pm 1 and 0 , respectively. The $L_{0}$ splitting reduces the confining gap to $E_{0^{-}}-E_{-1} \approx 50$ meV. First states crossing $E_{\mathrm{F}}$ from $\mathrm{LL}_{ \pm 1}$ are highlighted in orange. Uncertainty in $E_{\mathrm{F}}$ indicated as blue horizontal bar (Supporting Information). The green rectangle marks the zoom shown in panel e. $(b-d)$ Color plot of the wave function amplitude $|\Psi|=\sqrt{\left|\psi_{A}\right|^{2}+\left|\psi_{B}\right|^{2}}$ of states marked by orange crosses in panel e. $\Phi_{0}^{\mathrm{el}}$ at the crossing point $\varepsilon_{l}\left(\Phi_{0}^{\mathrm{el}}\right)=E_{\mathrm{F}}$ is marked. Solid (dashed) white lines are line cuts along the dotted white line in panel $\mathrm{b}$ for contributions from sublattice A (B), as marked. All scale bars identical. (e) Zoom into area marked by a green box in panel a. Colored lines identify valley $\mathrm{K}$ (cyan) and $\mathrm{K}^{\prime}$ (purple). Orange crosses mark crossing of $E_{\mathrm{F}}$ (blue dashed line) of selected states, which are displayed in panels $\mathrm{b}-\mathrm{d}$. First two orbital $\Delta_{j}^{o}$ and valley $\Delta_{k}^{\tau}$ splittings marked by arrows. (f) Comparison of length scales: tip-induced potential, magenta; calculated wave function amplitude $|\Psi|$ of first state crossing $E_{\mathrm{F}}$ (same as panel b) for sublattice A (gray line) and B (dashed line); superstructure lattice constant $a=13.8 \mathrm{~nm}$; magnetic length $l_{\mathrm{B}}(7 \mathrm{~T})=9.7 \mathrm{~nm}$.

$4 \mathrm{~b}$ portray $(0,0)$ and $(0,1)$ on sublattice $A$ and $B$, respectively. As expected, small angular momentum states are the first ones to localize with increasing $\Phi_{0}^{\mathrm{el}}$, which is in line with calculations by Giavaras et al. ${ }^{30}$ Notice that the applied $B$ naturally lifts the orbital degeneracy in QDs. ${ }^{57}$ Delocalized states remain at bulk LL energies (red horizontal lines in Figure 4a).

We distinguish two regimes in the sequence of spin degenerate states crossing $E_{\mathrm{F}}$ for negative $\Phi_{0}^{\mathrm{el}}$. The first regime (Figure 4e) exhibits $\Delta_{k}^{\tau} \lesssim \Delta_{j}^{\mathrm{o}} \lesssim E_{\mathrm{C}}^{i}$, while the second at higher $\Phi_{0}^{\mathrm{el}}$ is characterized by densely spaced states, thus $\Delta_{j}^{\mathrm{o}} \approx \Delta_{k}^{\tau} \ll$ $E_{\mathrm{C}}^{i}$. The sequence within the first regime corresponds to about five orbital pairs from valley $\mathrm{K}$ and $\mathrm{K}^{\prime}$, which is in line with about five quadruplets in our experimental spectra (see labels " 4 " in Figure $2 \mathrm{a}$ and $\Delta V$ sequences in Figure $2 \mathrm{~b}, \mathrm{c}$ ). The quite uniform spacing of peaks for larger $V_{\text {tip }}$ (Figure 2a) agrees with the second regime. In order to extract $\Delta_{j}^{o}$ and $\Delta_{k}^{\tau}$ within the first regime, we carefully assign the valley index to the states. Using the previously determined $n_{\mathrm{r}}$ and $m$ in eq 3 , the first state crossing $E_{\mathrm{F}}$ (Figure $4 \mathrm{~b}$ ) features LL index $N_{\mathrm{A}}=0+1 / 2(0+|0|)$ $=0$ on sublattice $\mathrm{A}$ and $N_{\mathrm{B}}=1+1 / 2(0+|0|)=1$ on sublattice $\mathrm{B}$, as predicted by eq 2 for a $\mathrm{LL}_{|1|}$ state in valley $\mathrm{K}$. The role of the sublattices interchanges for the second state crossing $E_{\mathrm{F}}$ (Figure $4 \mathrm{c}$ ), placing it in valley $\mathrm{K}^{\prime}$. Consequently, states with $N_{\mathrm{A}}=N_{\mathrm{B}}-1$ and $N_{\mathrm{B}}=N_{\mathrm{A}}-1$ are assigned to valleys $\mathrm{K}$ and $\mathrm{K}^{\prime}$, respectively. The calculation therefore predicts a valley splitting of about $\Delta_{1}^{\tau}=3 \mathrm{meV}$ on the $\mathrm{AB}$ and $\mathrm{BA}$ areas (see Figure $4 \mathrm{~b}, \mathrm{c}, \mathrm{e}) . \Delta_{2}^{\tau}$ is comparatively large (about $12 \mathrm{meV}$ ) and the respective orbital splitting $\Delta_{2}^{\mathrm{o}}$ is only larger by $1-2 \mathrm{meV}$ (see Figure 4e). Consequently, additional electrons may occupy the next orbital state of one valley prior to the same orbital state of the other valley at higher occupation numbers. Hence, we limit further comparison to experiment to $\Delta_{1}^{\tau}$. In our TB model, the strength of the valley splitting is dominated by the sublattice symmetry breaking term due to the $\mathrm{BN}$ substrate. ${ }^{39}$ The calculations also show that the radial extent of the wave functions grows for the first couple of states crossing $E_{\mathrm{F}}$, as expected for increasing $m$ (compare Figure 4 panels $\mathrm{b}$ and $\mathrm{c}$ to panel $\mathrm{d}$ ), explaining the decrease of $E_{\mathrm{C}}^{i}$ toward higher peak indices at fixed $B$ (see Figure $2 b, c)$.

Theory and experiment can be directly compared for the $B$ dependence of the onset voltage of charging peaks $V^{*}$. Experimentally, $V^{*}$ shifts toward higher $\left|V_{\text {tip }}\right|$ for increasing $B$ (Figure $5 \mathrm{a}$ ), thus gating the first state to $E_{\mathrm{F}}$ requires stronger band bending for higher $B$. Because the curves for $B>0 \mathrm{~T}$ are offset proportional to $\sqrt{ } B$, the straight line connecting the first charging peaks reveals that the energy distance of the first state to $E_{\mathrm{F}}$ scales with $\sqrt{ } B$. This corresponds to the increase in bulk LL energies for $N \neq 0$ (eq 1 ), strongly suggesting those LLs as source of the confined states. This analysis is confirmed by our TB calculations, as the first crossing points of $\mathrm{LL}_{+1}$ states with the Fermi level $\Phi_{0}^{*}$ also shift toward higher $\left|\Phi_{0}^{\mathrm{el}}\right|$ with increasing B (Figure 5b). While the evolution of states with $\Phi_{\mathrm{gr}}^{\mathrm{el}}$ in Figure 4a is (approximately) symmetric with respect to $\Phi_{\mathrm{gr}}^{\mathrm{e}}$ $\rightarrow-\Phi_{\mathrm{gr}}^{\mathrm{el}}$, the previously discussed p-doping induces an asymmetry in $\Phi_{0}^{*}$ for electrons and holes (see the lines highlighted in orange in Figure 4a) and thus accounts for the observed asymmetry in $V^{*}$. In Figure $5 \mathrm{c}$, we compare $V^{*}$ and $\Phi_{0}^{*}$ by using the $\Phi_{0}^{\mathrm{el}}\left(V_{\text {tip }}\right)$ dependence from the Poisson solver (see inset Figure 5c, Supporting Information). Care must be taken to correctly account for the work function difference between the tip and the sample: the tip's work function (4.5$\left.4.8 \mathrm{eV}^{36,58}\right)$ exceeds that of graphene $(4.5 \mathrm{eV})$, placing electric field neutrality in the positive $V_{\text {tip }}$ sector. Moreover, it definitely has to lie in between the two charging peak regimes because the QD vanishes without band bending. Using a plausible work function difference of $+50 \mathrm{meV}$ in Figure $5 \mathrm{c}$ leads to satisfactory agreement between the theoretical predictions for the first state crossings and the experimental $V^{*}$. 

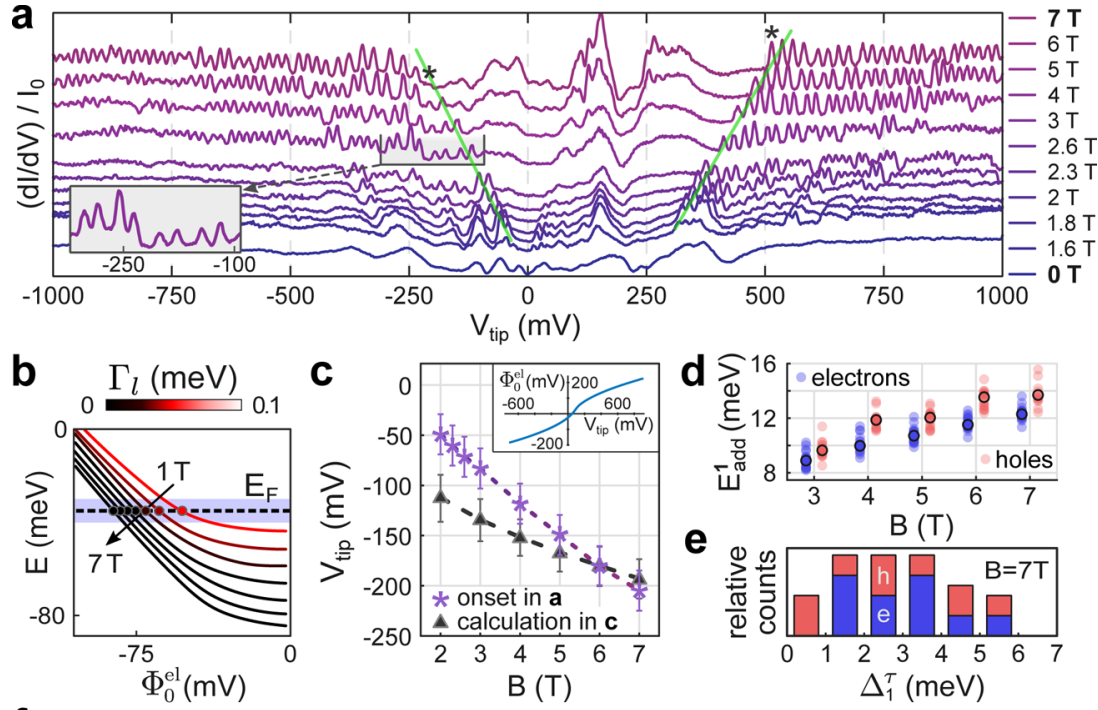

$$
\text { e }
$$
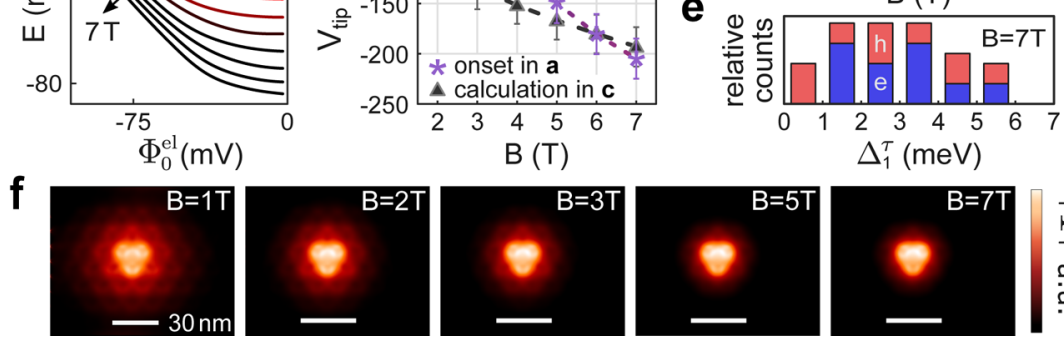

Figure 5. (a) $\mathrm{d} I / \mathrm{d} V$ spectroscopy in the vicinity of an AA stacked area at varying $B$, marked on the right. Four spatially adjacent spectra are averaged and the ones for $B>0 \mathrm{~T}$ are offset by a value proportional to $\sqrt{B} . V_{\text {stab }}=1000 \mathrm{mV}, I_{\text {stab }}=700 \mathrm{pA}, V_{\text {mod }}=4.2 \mathrm{mV}_{\text {rms. }}$ Green lines are guides to the eye, marking the onset voltage of charging peaks $V^{*}$. At $7 \mathrm{~T}$, an asterisk marks the first charging peak on either side. Inset shows zoom onto marked peaks. (b) Energy of first confined hole state as a function of induced potential amplitude $\Phi_{0}^{\text {el }}$ for different $B$ as marked. At larger $B$, states cross $E_{\mathrm{F}}$ at larger $\Phi_{0}^{\mathrm{el}}$, shifting $V^{*}$ to larger negative $V_{\text {tip. }}$. Color codes imaginary part of the eigenenergy as in Figure 4a. (c) Comparison between measured and calculated $V^{*}$. Inset shows the required $\Phi_{0}^{\mathrm{el}}\left(V_{\text {tip }}\right)$ for conversion, taken from a Poisson-solver (Supporting Information) and including a reasonable work function difference of $\Delta \Phi=50 \mathrm{meV}$ between graphene and tip. Error bars for measured $V^{*}$ reflect typical variation of $V^{*}$ on AA areas across a few superstructure unit cells. Error bars for calculation arise from the uncertainty in $E_{\mathrm{F}}$. (d) Plot of the $B$ dependence of $E_{\text {add }}^{1} \approx E_{\mathrm{C}}^{1}$ of 20 spectra (semitransparent dots) in the vicinity of an AA area. Data points are recorded at integer valued $B$ fields (in Tesla) but displayed slightly shifted to the left (electrons, blue) and to the right (holes, red) for clarity. Median values are encircled in black. (e) Histogram of $\Delta_{1}^{\tau} \approx E_{\text {add }}^{2}-E_{\text {add }}^{3}$ (experimental error below $0.2 \mathrm{meV}$ ) at $B=7 \mathrm{~T}$ for the same AA area used in panel d. Electron (blue bars) and hole (red bars) contributions are colored. (f) Calculated $|\Psi|$ of the first confined hole state (see panel b) crossing $E_{\mathrm{F}}$ at different $B$ as marked. The state originates from $\mathrm{LL}_{-1}$, i.e., $V_{\text {tip }}<0 \mathrm{~V}$ when crossing. All scale bars identical.

Our TB simulations predict a strong reduction of $\Gamma_{l}$ with increasing magnetic field, corresponding to the suppression of the radial tail of the wave function in Figure $5 \mathrm{f}$ and indicating the onset of localization between 1 and $3 \mathrm{~T}$ (Figure $5 \mathrm{~b}$ ). The first appearance of charging peaks in the experiment at around $2 \mathrm{~T}$ (Figure 5a) fits nicely. This finding is further corroborated by comparing the diameter of the LL state $d_{n}=2 \sqrt{2 n+1} \cdot l_{\mathrm{B}}$, being $d_{1}=89 \mathrm{~nm}(63 \mathrm{~nm})$ for $\mathrm{LL}_{1}$ at $1 \mathrm{~T}(2 \mathrm{~T})$ with the fwhm of the band bending region of $55 \mathrm{~nm}$, providing an independent confirmation of the estimated $\Phi_{\mathrm{gr}}^{\mathrm{el}}$. At higher $B$, the diameter of the first QD state wave function is dominated by $l_{\mathrm{B}}$ rather than by the width of $\Phi_{\mathrm{gr}}^{\mathrm{el}}$ (Figure $4 \mathrm{f}$ ). The compression of the wave function for increasing $B$ (Figure 5f) also manifests itself as increase in addition energy, for instance, for $E_{\text {add }}^{1}=E_{\mathrm{C}}^{1}+\Delta^{\sigma}$ in Figure $5 \mathrm{~d}$, where the increase in $E_{\text {add }}^{1}$ with $B$ by about $4 \mathrm{meV}$ cannot be explained by that of $\Delta^{\sigma}$, being $460 \mu \mathrm{eV}$ between 3 and $7 \mathrm{~T}$. Consequently, increased Coulomb repulsion between electrons due to stronger compression and thus larger $E_{\mathrm{C}}^{1}$ dominates $E_{\text {add }}^{1}(B)$. We observe a similar monotonic increase for the other $E_{\text {add }}^{i}$ with odd index $i$, independent of the position of the QD.

Experiment and theory also provide detailed insight into the valley splitting $\Delta_{k}^{\tau}$ of the first confined states. The peaks of the first quadruplets in Figures 2a and 5a (see, e.g., inset) often group in doublets, suggesting sizable values of either $\Delta_{k}^{\tau}$ or $\Delta^{\sigma}$ (Figure 1e,f). While $\Delta^{\sigma}$ is expected to be spatially homogeneous and only weakly varying between different quadruplets, the TB calculations predict strongly varying $\Delta_{k}^{\tau}$ for different quadruplets (Figure $4 \mathrm{e}$ ), which is in accordance with our observations in the experimental spectra. For a quantitative comparison, we focus on $E_{\text {add }}^{2}$ which separates the two doublets within the first quadruplet. In view of the small value of the Zeeman splitting $\left(\Delta^{\sigma} \approx 800 \mu \mathrm{eV}\right.$ at $\left.7 \mathrm{~T}\right)$, we approximate $E_{\mathrm{C}}^{2}$ by $E_{\text {add }}^{3}$ to extract the valley splitting $\Delta_{1}^{\tau} \approx E_{\text {add }}^{2}$ $-E_{\text {add }}^{3}$. We record 20 spectra in the vicinity of an AA stacked area at $B=7 \mathrm{~T}$ to obtain a histogram of $\Delta_{1}^{\tau}$ for electrons and holes (Figure 5e), where $\Delta_{1}^{\tau}$ could be determined with an experimental error smaller than $0.2 \mathrm{meV}$. The values strikingly group around the predicted $\Delta_{1}^{\tau} \approx 3 \mathrm{meV}$ found in the TB calculations (Figure 4e), with a probable offset in the QD position relative to the tunneling tip (Supporting Information, Section 5) explaining the QD probing an area adjacent to the tunneling tip. We conclude that sizable $\Delta_{k}^{\tau}$ separate quadruplets into doublets, while the smaller $\Delta^{\sigma}$ contributes to the odd addition energies within the doublets. Realizing such a controlled lifting of one of the two degeneracies in graphene QDs is a key requirement for 2-qubit gate operation. ${ }^{2}$ It enables Pauli blockade in exchange driven qubits as required for scalable quantum computation approaches using graphene. ${ }^{2}$ Our observation of valley splittings, so far elusive, provides a stepping stone toward the exploitation of the presumably large coherence time of electron spins in graphene QDs. ${ }^{2-5}$ 
In summary, we have realized graphene quantum dots without physical edges via electrostatic confinement in magnetic field using low disorder graphene crystallographically aligned to a hexagonal boron nitride substrate. We observe more than 40 charging peaks in the hole and electron sector arranged in quadruplets due to orbital splittings. The first few peaks on the hole and electron side show an additional doublet structure traced back to lifting of the valley degeneracy. Note that such a lifting is key for the use of graphene quantum dots as spin qubits. ${ }^{2}$ Tight binding calculations quantitatively reproduce the orbital splitting energy of $4-10 \mathrm{meV}$ as well as the first orbital's valley splitting energy of about $3 \mathrm{meV}$ by assuming a tip potential deduced from an electrostatic Poisson calculation. Also the onset of confinement at about $2 \mathrm{~T}$ is well reproduced by the calculation. Our results demonstrate a much better controlled confinement by combining magnetic and electrostatic fields than previously found in graphene. Exploiting the present approach in transport merely requires replacing the tip by a conventional electrostatic gate with a diameter of about $100 \mathrm{~nm}$. Moreover, the approach allows for straightforward tuning of (i) orbital splittings by changing the gate geometry and thus the confinement potential, (ii) valley splittings based on substrate interaction, (iii) the Zeeman splitting by altering the magnetic field, and (iv) the coupling of dot states to leads or to other quantum dots by changing the magnetic field or selecting a different quantum dot state. Finally, our novel mobile quantum dot enables a detailed investigation of structural details of graphene stacked on various substrates, by spatially mapping the quantum dot energies.

\section{ASSOCIATED CONTENT}

\section{S Supporting Information}

The Supporting Information is available free of charge on the ACS Publications website at DOI: 10.1021/acs.nanolett.6b02548.

Sample preparation; STM measurement details; Poisson solver; capacitances, tip lever arm and tunnel-coupling of QD states; Landau levels and estimation of the Fermi energy; identication of defect charging peaks; assigning a valley to the TB wave functions; additional references and figures (PDF)

\section{AUTHOR INFORMATION}

\section{Corresponding Author}

*E-mail: MMorgens@physik.rwth-aachen.de. Fax: 004924180 22306.

\section{Notes}

The authors declare no competing financial interest.

\section{ACKNOWLEDGMENTS}

The authors thank C. Stampfer, R. Bindel, M. Liebmann and K. Flöhr for prolific discussions, as well as C. Holl for contributions to the Poisson calculations and A. Georgi for assisting the measurements. N.M.F., P.N., and M.M. gratefully acknowledge support from the Graphene Flagship (Contract No. NECTICT-604391) and the German Science foundation (Li 1050-2/2 through SPP-1459). L.A.C., J.B., and F.L. from the Austrian Fonds zur Förderung der wissenschaftlichen Forschung (FWF) through the SFB 041-ViCom and doctoral college Solids4Fun (W1243). Calculations were performed on the Vienna Scientific Cluster. R.V.G., A.K.G., and K.S.N. also acknowledge support from EPSRC (Towards Engineering Grand Challenges and Fellowship programs), the Royal Society, U.S. Army Research Office, U.S. Navy Research Office, and U.S. Airforce Research Office. K.S.N. is also grateful to ERC for support via Synergy grant Hetero2D. A.K.G. was supported by Lloyd's Register Foundation.

\section{REFERENCES}

(1) Castro Neto, A. H.; Guinea, F.; Peres, N. M. R.; Novoselov, K. S.; Geim, A. K. Rev. Mod. Phys. 2009, 81, 109-162.

(2) Trauzettel, B.; Bulaev, D. V.; Loss, D.; Burkard, G. Nat. Phys. 2007, 3, 192-196.

(3) Fuchs, M.; Rychkov, V.; Trauzettel, B. Phys. Rev. B: Condens. Matter Mater. Phys. 2012, 86, 085301.

(4) Droth, M.; Burkard, G. Phys. Rev. B: Condens. Matter Mater. Phys. 2013, 87, 205432.

(5) Fuchs, M.; Schliemann, J.; Trauzettel, B. Phys. Rev. B: Condens. Matter Mater. Phys. 2013, 88, 245441.

(6) Bischoff, D.; Varlet, A.; Simonet, P.; Eich, M.; Overweg, H. C.; Ihn, T.; Ensslin, K. Appl. Phys. Rev. 2015, 2, 031301.

(7) Giesbers, A. J. M.; Zeitler, U.; Neubeck, S.; Freitag, F.; Novoselov, K. S.; Maan, J. C. Solid State Commun. 2008, 147, 366369.

(8) Morgenstern, M.; Freitag, N.; Vaid, A.; Pratzer, M.; Liebmann, M. Phys. Status Solidi RRL 2016, 10, 24-38.

(9) Magda, G. Z.; Jin, X.; Hagymasi, I.; Vancso, P.; Osvath, Z.; Nemes-Incze, P.; Hwang, C.; Biro, L. P.; Tapaszto, L. Nature 2014, 514, 608-611.

(10) Liu, Z.; Ma, L.; Shi, G.; Zhou, W.; Gong, Y.; Lei, S.; Yang, X.; Zhang, J.; Yu, J.; Hackenberg, K. P.; Babakhani, A.; Idrobo, J. C.; Vajtai, R.; Lou, J.; Ajayan, P. M. Nat. Nanotechnol. 2013, 8, 119-124.

(11) Qi, Z. J.; Daniels, C.; Hong, S. J.; Park, Y. W.; Meunier, V.; Drndic, M.; Johnson, A. T. ACS Nano 2015, 9, 3510-3520.

(12) Vicarelli, L.; Heerema, S. J.; Dekker, C.; Zandbergen, H. W. ACS Nano 2015, 9, 3428-3435.

(13) Tarucha, S.; Austing, D. G.; Honda, T.; van der Hage, R. J.; Kouwenhoven, L. P. Phys. Rev. Lett. 1996, 77, 3613-3616.

(14) Kouwenhoven, L. P.; Austing, D. G.; Tarucha, S. Rep. Prog. Phys. 2001, 64, 701-736.

(15) Libisch, F.; Rotter, S.; Burgdörfer, J. New J. Phys. 2012, 14, 123006.

(16) Bischoff, D.; Simonet, P.; Varlet, A.; Overweg, H. C.; Eich, M.; Ihn, T.; Ensslin, K. Phys. Status Solidi RRL 2016, 10, 68-74.

(17) Terres, B.; Chizhova, L. A.; Libisch, F.; Peiro, J.; Jörger, D.; Engels, S.; Girschik, A.; Watanabe, K.; Taniguchi, T.; Rotkin, S. V.; Burgdörfer, J.; Stampfer, C. Nat. Commun. 2016, 7, 11528.

(18) Rycerz, A.; Tworzydlo, J.; Beenakker, C. W. J. Nat. Phys. 2007, 3, 172-175.

(19) Recher, P.; Nilsson, J.; Burkard, G.; Trauzettel, B. Phys. Rev. B: Condens. Matter Mater. Phys. 2009, 79, 085407.

(20) Zhang, Y. B.; Tang, T. T.; Girit, C.; Hao, Z.; Martin, M. C.; Zettl, A.; Crommie, M. F.; Shen, Y. R.; Wang, F. Nature 2009, 459, $820-823$.

(21) Allen, M. T.; Martin, J.; Yacoby, A. Nat. Commun. 2012, 3, 934. (22) Goossens, A. M.; Driessen, S. C. M.; Baart, T. A.; Watanabe, K.; Taniguchi, T.; Vandersypen, L. M. K. Nano Lett. 2012, 12, 46564660.

(23) Müller, A.; Kaestner, B.; Hohls, F.; Weimann, T.; Pierz, K.; Schumacher, H. W. J. Appl. Phys. 2014, 115, 233710.

(24) Ju, L.; Shi, Z. W.; Nair, N.; Lv, Y. C.; Jin, C. H.; Velasco, J., Jr; Ojeda-Aristizabal, C.; Bechtel, H. A.; Martin, M. C.; Zettl, A.; Analytis, J.; Wang, F. Nature 2015, 520, 650-655.

(25) Zhao, Y.; Wyrick, J.; Natterer, F. D.; Rodriguez-Nieva, J. F.; Lewandowski, C.; Watanabe, K.; Taniguchi, T.; Levitov, L. S.; Zhitenev, N. B.; Stroscio, J. A. Science 2015, 348, 672-675.

(26) Lee, J.; Wong, D.; Velasco, J., Jr; Rodriguez-Nieva, J. F.; Kahn, S.; Tsai, H.-Z.; Taniguchi, T.; Watanabe, K.; Zettl, A.; Wang, F.; 
Levitov, L. S.; Crommie, M. F. Nat. Phys. 2016, DOI: 10.1038/ nphys3805.

(27) Gutierrez, C.; Brown, L.; Kim, C.-J.; Park, J.; Pasupathy, A. N. Nat. Phys. 2016, DOI: $10.1038 /$ nphys3806.

(28) Klimov, N. N.; Jung, S.; Zhu, S.; Li, T.; Wright, C. A.; Solares, S. D.; Newell, D. B.; Zhitenev, N. B.; Stroscio, J. A. Science 2012, 336, 1557-1561.

(29) Chen, H. Y.; Apalkov, V.; Chakraborty, T. Phys. Rev. Lett. 2007, $98,186803$.

(30) Giavaras, G.; Maksym, P. A.; Roy, M. J. Phys.: Condens. Matter 2009, 21, 102201.

(31) Giavaras, G.; Nori, F. Phys. Rev. B: Condens. Matter Mater. Phys. 2012, 85, 165446

(32) Moriyama, S.; Morita, Y.; Watanabe, E.; Tsuya, D. Appl. Phys. Lett. 2014, 104, 053108.

(33) Jung, S. Y.; Rutter, G. M.; Klimov, N. N.; Newell, D. B.; Calizo, I.; Walker, A. R. H.; Zhitenev, N. B.; Stroscio, J. A. Nat. Phys. 2011, 7, 245-251.

(34) Luican-Mayer, A.; Kharitonov, M.; Li, G.; Lu, C. P.; Skachko, I.; Goncalves, A. M.; Watanabe, K.; Taniguchi, T.; Andrei, E. Y. Phys. Rev. Lett. 2014, 112, 036804.

(35) Tovari, E.; Makk, P.; Rickhaus, P.; Schönenberger, C.; Csonka, S. Nanoscale 2016, 8, 11480-11486.

(36) Dombrowski, R.; Steinebach, C.; Wittneven, C.; Morgenstern, M.; Wiesendanger, R. Phys. Rev. B: Condens. Matter Mater. Phys. 1999, 59, 8043-8048.

(37) Morgenstern, M.; Haude, D.; Gudmundsson, V.; Wittneven, C.; Dombrowski, R.; Wiesendanger, R. Phys. Rev. B: Condens. Matter Mater. Phys. 2000, 62, 7257-7263.

(38) Libisch, F.; Rotter, S.; Güttinger, J.; Stampfer, C.; Burgdörfer, J. Phys. Rev. B: Condens. Matter Mater. Phys. 2010, 81, 245411.

(39) Chizhova, L. A.; Libisch, F.; Burgdörfer, J. Phys. Rev. B: Condens. Matter Mater. Phys. 2014, 90, 165404.

(40) Averin, D. V.; Likharev, K. K. J. Low Temp. Phys. 1986, 62, 345373.

(41) Mayorov, A. S.; Gorbachev, R. V.; Morozov, S. V.; Britnell, L.; Jalil, R.; Ponomarenko, L. A.; Blake, P.; Novoselov, K. S.; Watanabe, K.; Taniguchi, T.; Geim, A. K. Nano Lett. 2011, 11, 2396-2399.

(42) Kretinin, A. V.; et al. Nano Lett. 2014, 14, 3270-3276.

(43) Gorbachev, R. V.; Song, J. C.; Yu, G. L.; Kretinin, A. V.; Withers, F.; Cao, Y.; Mishchenko, A.; Grigorieva, I. V.; Novoselov, K. S.; Levitov, L. S.; Geim, A. K. Science 2014, 346, 448-51.

(44) Hunt, B.; Sanchez-Yamagishi, J. D.; Young, A. F.; Yankowitz, M.; LeRoy, B. J.; Watanabe, K.; Taniguchi, T.; Moon, P.; Koshino, M.; Jarillo-Herrero, P.; Ashoori, R. C. Science 2013, 340, 1427-1430.

(45) Woods, C. R.; et al. Nat. Phys. 2014, 10, 451-456.

(46) Decker, R; Wang, Y.; Brar, V. W.; Regan, W.; Tsai, H. Z.; Wu, Q.; Gannett, W.; Zettl, A.; Crommie, M. F. Nano Lett. 2011, 11, 2291-2295.

(47) Xue, J. M.; Sanchez-Yamagishi, J.; Bulmash, D.; Jacquod, P.; Deshpande, A.; Watanabe, K.; Taniguchi, T.; Jarillo-Herrero, P.; LeRoy, B. J. Nat. Mater. 2011, 10, 282-285.

(48) Deshpande, A.; LeRoy, B. J. Phys. E 2012, 44, 743-759.

(49) Mashoff, T.; Pratzer, M.; Morgenstern, M. Rev. Sci. Instrum. 2009, 80, 053702 .

(50) Wong, D.; Velasco, J., Jr; Ju, L.; Lee, J.; Kahn, S.; Tsai, H. Z.; Germany, C.; Taniguchi, T.; Watanabe, K.; Zettl, A.; Wang, F.; Crommie, M. F. Nat. Nanotechnol. 2015, 10, 949-953.

(51) Hanna, A. E.; Tinkham, M. Phys. Rev. B: Condens. Matter Mater. Phys. 1991, 44, 5919-5922.

(52) Wildöer, J. W. G.; van Roij, A. J. A.; Harmans, C. J. P. M.; van Kempen, H. Phys. Rev. B: Condens. Matter Mater. Phys. 1996, 53, 10695-10698.

(53) Schnez, S.; Ensslin, K.; Sigrist, M.; Ihn, T. Phys. Rev. B: Condens. Matter Mater. Phys. 2008, 78, 195427. Yoshioka, D. J. Phys. Soc. Jpn. 2007, 76, 024718.

(54) Sachs, B.; Wehling, T. O.; Katsnelson, M. I.; Lichtenstein, A. I. Phys. Rev. B: Condens. Matter Mater. Phys. 2011, 84, 195414.
(55) Martinez-Gordillo, R.; Roche, S.; Ortmann, F.; Pruneda, M. Phys. Rev. B: Condens. Matter Mater. Phys. 2014, 89, 161401.

(56) Bischoff, D.; Libisch, F.; Burgdörfer, J.; Ihn, T.; Ensslin, K. Phys. Rev. B: Condens. Matter Mater. Phys. 2014, 90, 115405.

(57) Fock, V. Eur. Phys. J. A 1928, 47, 446-448.

(58) Chen, Y. C.; Zhao, C. C.; Huang, F.; Zhan, R. Z.; Deng, S. Z.; Xu, N. S.; Chen, J. Sci. Rep. 2016, 6, 21270. 